\title{
Comparing diet and exercise
}

It is common knowledge that diet and exercise are key to achieving and maintaining a healthy weight, but many people have their own opinions about which factor is most important. And it is a challenge to disentangle each factor's respective effects, as sedentary behavior is often associated with excess caloric intake.

A team of researchers lead by Vicki Vieira-Potter (University of Missouri, Columbia) aimed to compare these two attributes and their effects, finding that exercise brought about a complex suite of metabolic changes that do not occur during comparable caloric restriction in sedentary animals (Med. Sci. Sports Exerc. doi:10.1249/MSS.0000000000000964; published online 29 April 2016). They carried out this study using obesity-prone rats, all of which received a high-fat 'Western'-style diet. These were divided into three groups with different diet and exercise treatments: 'sedentary', with no access to exercise and ad libitum access to food; 'exercise', with a voluntary running wheel and ad libitum access to food; 'weight-matched', with no access exercise and restricted access to food based on daily weight comparisons against the exercise group.

After several weeks, Vieira-Potter's team evaluated a variety of metabolic variables and measured the gut microbial communities of each group. Both exercise and caloric restriction improved body composition and reduced inflammation of adipose tissues and aorta, but exercise also produced several other beneficial effects, including increased insulin sensitivity and enhanced fat oxidation. Curiously, microbial composition in the gut appeared to be differentially affected by both exercise and restricted food intake, and some of the observed changes correlated with positive metabolic changes that were noted in the exercise group. There were also behavioral differences among the groups. "Overall, the exercising rats had higher metabolic rates, were more active even when not running on their wheels and experienced shifts in their gut microbes," Vieira-Potter summarized in a press release, suggesting that this could put the exercising rodents "in a better

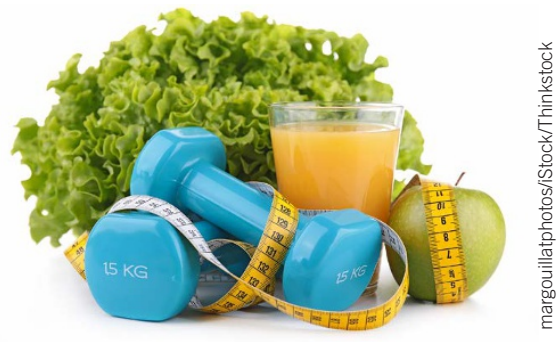

position to avoid future weight gain compared to the other groups."

These findings reveal the importance of exercise in weight loss and, more generally, a healthy lifestyle. By identifying the precise changes that take place, this study also identifies many potential causes for the dysfunctions that are associated with sedentary lifestyles. Vieira-Potter and her coauthors are careful to note, however, that the mechanisms and isolated consequences of these microbial and metabolic changes still require further investigation. Nonetheless, this research adds convincing evidence to the case of exercise as a primary mediator of weight and metabolism.

Gregory D. Larsen

\section{BEETLES MODEL CONFUSING COPULATIONS}

Same-sex sexual behaviors, which occur in various animal species, present a curious conundrum for evolutionary biologists: often these behaviors are energetically expensive, but they yield no obvious benefits to an individual's fitness. One explanatory theory is that such behaviors arise from sexual conflict, which occurs when an optimal fitness strategy in one sex causes counterproductive effects in the other sex. Sometimes this sexual conflict concerns a specific locus whose expression affects fitness differentially between males and females; this is called intralocus sexual conflict. It is possible that, at least in some cases, same-sex sexual behaviors arise through intralocus sexual conflict as a side-effect of other beneficial adaptations that outweigh the costs of ineffectual intercourse.

David Berger and colleagues at Uppsala University (Sweden) established that this is the case, for instance, in the seed beetle Callosobruchus maculatus, which conveniently displays same-sex sexual behaviors when housed in same-sex groups. Using artificial selection, Berger's team bred genetic strains of $C$. maculatus that showed an increased proclivity for same-sex sexual behavior (BMC Evol. Biol. 16, 88; 2016). Using constructed scenarios in Petri dish arenas, the team then evaluated various attributes of the beetles, such as perception and locomotor activity, in males, and lifetime reproductive success of each sex.

The results were complex, showing that same-sex sexual behavior was associated with different outcomes in each sex. "For example," Berger explains, "we noted that males that had been bred for increased same-sex mounting behavior were less discriminating when given a choice between courting a male or a female in later tests, while their sisters laid more eggs and produced more offspring than before." Other differences were weak or not statistically significant, but generally indicated that the genetic basis for same-sex sexual behavior in these beetles is associated with both shared and sex-limited effects. This might not be surprising, given how often phenotypes are governed by multiple genes, and how often pleiotropic genes influence multiple phenotypes, but it does demonstrate how same-sex sexual behavior among seed beetles, and perhaps other insects, is integrated with a suite of important attributes and genetic influences. Such complexity lends support to the theory that same-sex sexual behaviors can arise from sexual conflict, when selective pressures promote related phenotypes that also influence reproductive success.

While such findings cannot be generalized to other species-indeed, they have limited application even to wild, naturally selected populations-they demonstrate a plausible evolutionary mechanism by which this reproductively inert behavior might arise naturally.

Gregory D. Larsen 